\title{
ON SUBSEQUENTIAL LIMIT POINTS OF A SEQUENCE OF ITERATES. II
}

\author{
M. MAITI and A. C. BABU
}

(Received March 26, 1983)

Communicated by J. M. Rubinstein

\begin{abstract}
J. B. Diaz and F. T. Metcalf established some results concerning the structure of the set of cluster points of a sequence of iterates of a continuous self-map of a metric space. In this paper it is shown that their conclusions remain valid if the distance function in their inequality is replaced by a continuous function on the product space. Then this idea is extended to some other mappings and to uniform and general topological spaces.
\end{abstract}

1980 Mathematics subject classification (Amer. Math. Soc.): 47 H 10, 54 H 25.

Diaz and Metcalf $[5,6]$ have studied the structure of the set of subsequential limit points of a sequence of iterates of a continuous self-map $A$ of a metric space $(X, d)$ satisfying the condition $d(A x, F(A))<d(x, F(A))$, where $x \neq A x$ and $F(A)$, the set of fixed points of $A$, is nonempty and compact. In this paper it is shown that the conclusions of Diaz and Metcalf [6] may still be derived after replacing $d$ by a continuous function $\varphi: X \times X \rightarrow R_{0}$, where $R_{0}$ is the subspace $[0, \infty)$ of the real line with usual topology. Then our analysis is extended to the mappings introduced by Dotson [7], Browder and Petryshyn [2, 3], Singh and Zorzitto [9] and Caristi [4]. Then we show that our results may be carried over to uniform spaces and, further, that some of our conclusions hold in Hausdorff topological spaces.

In what follows $\varphi(x, F(A))$ will be used to denote inf ${ }_{y \in F(A)}(x, y)$. The orbit of $x \in X$ generated by $A$ will be denoted by $O(x, A)$ and its closure by $\bar{O}(x, A)$. The set of subsequential limit points of the sequence $\left\{A^{n} x\right\}_{n=0}^{\infty}$ will be denoted by $\mathscr{L}(x)$.

(c) 1985 Australian Mathematical Society $0263-6115 / 85 \$ A 2.00+0.00$ 
We now establish the generalization of Theorem 2 of Diaz and Metcalf [6].

THEOREM 1. Let $A$ be a continuous self-map of a metric space $(X, d)$. Suppose that

(i) $F(A)$ is nonempty and compact.

(ii) there exists a non-negative continuous function $\varphi: X \times X \rightarrow R_{0}$ such that $\varphi(A y, F(A))<\varphi(y, F(A))$ for $y \in X-F(A)$,

(iii) $\bar{O}(x, A)$ is compact for some $x \in X$ :

Then $\mathscr{L}(x)$ is a nonempty, compact and connected subset of $F(A)$. Either $\mathscr{L}(x)$ is a singleton or is uncountable. In the case $\mathscr{L}(x)$ is a singleton, $\lim _{n \rightarrow \infty} A^{n} x$ exists and belongs to $F(A)$. In the case $\mathscr{L}(x)$ is uncountable, it is contained in the boundary of $F(A)$.

Proof. The compactness of $\bar{O}(x, A)$ implies nonemptiness of $\mathscr{L}(x)$. We now show that $\mathscr{L}(x) \subset F(A)$. If some iterate $A^{k} x \in F(A)$, we have $\mathscr{L}(x)=\left\{A^{k} x\right\} \subset$ $F(A)$ and the theorem is proved. Therefore we assume that $A^{k} x \notin F(A)$ for $k=0,1,2, \ldots$. Since for any fixed $y, z \rightarrow \varphi(z, y)$ is a continuous function from $X \rightarrow R_{0}$, the function $z \rightarrow \varphi(z, F(A))$ is an upper semi-continuous function, being the infimum of a family of continuous functions. Since $A^{k} x \notin F(A)$ for all $k$, we have $\varphi\left(A^{k+1} x, F(A)\right)<\varphi\left(A^{k} x, F(A)\right)$ for all $k$. Therefore $\left\{\varphi\left(A^{k} x, F(A)\right)\right\}_{k=0}^{\infty}$ is a monotonically decreasing sequence of nonnegative real numbers and so will converge to $r \geqslant 0$, say. Since $\mathscr{L}(x) \neq \varnothing$, for a $\xi \in \mathscr{L}(x)$ there exists a subsequence $\left\{A^{n_{i} x}\right\}_{i=1}^{\infty}$ with $A^{n_{i} x} \rightarrow \xi$ as $i \rightarrow \infty$. If $A \xi=\xi$, then we are through. Therefore we assume that $\xi \neq A \xi$. Then $r=\lim \varphi\left(A^{1+n_{i}} x, F(A)\right)=$ $\lim \sup \varphi\left(A^{1+n_{i}} x, F(A)\right) \leqslant \varphi\left(\lim A^{1+n_{i}} x, F(A)\right)=\varphi(A \xi, \quad F(A))<\varphi(\xi, F(A))$. Now $y \rightarrow \varphi\left(A^{n} x, y\right)$, for fixed $A^{n} x$, is a continuous function: $X \rightarrow R_{0}$ and so will attain its infimum on $F(A)$. Therefore there exists a $p_{n} \in F(A)$ such that $\varphi\left(A^{n} x, F(A)\right)=\varphi\left(A^{n} x, p_{n}\right)$. Corresponding to each $A^{n_{i}} x$ of the convergent subsequence $\left\{A^{n_{i}} x\right\}_{i=1}^{\infty}$ we have a $p_{n_{i}} \in F(A)$. Since $F(A)$ is compact, $\left\{p_{n_{i}}\right\}_{i=1}^{\infty}$ will have a convergent subsequence denoted by $\left\{p_{m_{i}}\right\}_{i=1}^{\infty}$ converging to $q$, say, in $F(A)$. Now $A^{m_{i} x} \rightarrow q$. From $\varphi\left(A^{n+1} x, p_{n+1}\right)=\varphi\left(A^{n+1} x, F(A)\right)<$ $\varphi\left(A^{n} x, F(A)\right)=\varphi\left(A^{n} x, p_{n}\right)$, we have, for $m_{i}>n, \varphi\left(A^{m_{i}} x, p_{m_{i}}\right)<\varphi\left(A^{n} x, p_{n}\right)$. Letting $m_{i} \rightarrow \infty$, we have, since $\varphi$ is continuous, $\varphi(\xi, q)<\varphi\left(A^{n} x, p_{n}\right)$. Since $\varphi\left(A^{n} x, p_{n}\right)=\varphi\left(A^{n} x, F(A)\right) \rightarrow r \geqslant 0$, we have $\varphi(\xi, q) \leqslant r$. Thus $r \leqslant$ $\varphi(A \xi, F(A))<\varphi(\xi, F(A)) \leqslant \varphi(\xi, q) \leqslant r$. This is absurd. Therefore $\xi=A \xi$, that is, $\xi \in F(A)$. In other words $\mathscr{L}(x) \subset F(A)$. Further, $\mathscr{L}(x)$, being a closed subset of the compact set $F(A)$, is compact.

We now prove that $\mathscr{L}(x)$ is connected. Suppose the contrary. Then there exist two nonempty, disjoint, closed subsets $S_{1}, S_{2}$ of $\mathscr{L}(x)$ such that $\mathscr{L}(x)=S_{1} \cup S_{2}$. 
Since $S_{1}$ and $S_{2}$ are closed subsets of a compact set $\mathscr{L}(x)$, they are themselves compact. Hence $d\left(S_{1}, S_{2}\right)>0$. Next, we show that $d\left(A^{m} x, F(A)\right) \rightarrow 0$ as $m \rightarrow \infty$. If not, there exists an $\varepsilon>0$ and a subsequence $\left\{A^{m_{i}} x\right\}_{i=1}^{\infty}$ such that $d\left(A^{m_{i}} x, F(A)\right) \geqslant \varepsilon>0$ for $i=1,2,3, \ldots$ Since $\bar{O}(x, A)$ is compact, $\left\{A^{m_{i}} x\right\}_{i=1}^{\infty}$ will have a subsequence $\left\{A^{n_{i}} x\right\}_{i=1}^{\infty} \rightarrow \xi \in F(A)$. Thus $d\left(A^{n_{i}} x, F(A)\right) \leqslant$ $d\left(A^{n_{i}} x, \xi\right) \rightarrow 0$ as $i \rightarrow \infty$. This is a contradiction. Therefore we must have $d\left(A^{m} x, F(A)\right) \rightarrow 0$ as $m \rightarrow \infty$. We next prove that $\lim _{m \rightarrow \infty} d\left(A^{m} x, S_{1} \cup S_{2}\right)=0$. If it is not so, then there will exist an $\varepsilon>0$ and a subsequence $\left\{A^{m_{i}} x\right\}_{i=1}^{\infty}$ such that $d\left(A^{m_{i}} x, S_{1} \cup S_{2}\right) \geqslant \varepsilon>0$ for $i=1,2,3, \ldots$ Since $F(A)$ is compact, there exists a $q_{m_{i}} \in F(A)$ such that $d\left(A^{m_{i}} x, F(A)\right)=d\left(A^{m_{i}} x, q_{m_{i}}\right)$. Because of the compactness of $F(A),\left\{q_{m_{i}}\right\}_{i=1}^{\infty}$ will have a convergent subsequence $\left\{q_{n_{i}}\right\}_{i=1}^{\infty}$ with $q_{n_{i}} \rightarrow q \in F(A)$. Now $d\left(A^{n_{i}} x, q\right) \leqslant d\left(A^{n_{i}} x, q_{n_{i}}\right)+d\left(q_{n_{i}}, q\right) \rightarrow 0$. Hence $q \in$ $\mathscr{L}(x)=S_{1} \cup S_{2}$ and $d\left(A^{n_{i}} x, S_{1} \cup S_{2}\right) \leqslant d\left(A^{n_{i}} x, q\right) \rightarrow 0$ as $i \rightarrow \infty$. This contradiction shows that $\lim _{m \rightarrow \infty} d\left(A^{m} x, S_{1} \cup S_{2}\right)=0$. We further prove that $A$ is asymptotically regular. If not, there will exist an $\varepsilon>0$ and a subsequence $\left\{A^{m_{i}} x\right\}_{i=1}^{\infty}$ such that $d\left(A^{m_{i}} x, A^{1+m_{i}} x\right) \geqslant \varepsilon>0$. The corresponding sequence $\left\{q_{m_{i}}\right\}_{i=1}^{\infty}$ in $F(A)$ will have a subsequence $\left\{q_{n_{i}}\right\}_{i=1}^{\infty}$ converging to $q \in F(A)$. As above $A^{n_{i}} x \rightarrow q \in F(A)$. Since $A$ is continuous at $q$, we have $A^{1+n_{i}} x \rightarrow A_{q}=q$. Now $d\left(A^{n_{i}} x, A^{1+n_{i}} x\right) \leqslant d\left(A^{n_{i}} x, q\right)+d\left(A^{1+n_{i}} x, q\right) \rightarrow 0$ as $i \rightarrow \infty$. This is contrary to hypothesis. Hence we have proved that $d\left(A^{m} x, A^{m+1} x\right) \rightarrow 0$ as $i \rightarrow \infty$. Thus from the results found in this paragraph, we can find an integer $M$ such that for $m \geqslant M, d\left(A^{m} x, A^{m+1} x\right)<\frac{1}{3} d\left(S_{1}, S_{2}\right)$ and $d\left(A^{m} x, S_{1}, S_{2}\right)<\frac{1}{3} d\left(S_{1}, S_{2}\right)$. Since $S_{1} \cup S_{2}$ is compact there exists a $q \in S_{1} \cup S_{2}$ such that $d\left(A^{m} x, S_{1} \cup S_{2}\right)=$ $d\left(A^{m} x, q\right)$. If $q \in S_{1}$, then $d\left(A^{m} x, S_{1}\right) \leqslant d\left(A^{m} x, q\right)<\frac{1}{3} d\left(S_{1}, S_{2}\right)$. Therefore for any $m \geqslant M$, either $d\left(A^{m} x, S_{1}\right)<\frac{1}{3} d\left(S_{1}, S_{2}\right)$ or, $d\left(A^{m} x, S_{2}\right)<\frac{1}{3} d\left(S_{1}, S_{2}\right)$. Both these inequalities cannot hold simultaneously, because in that case $d\left(S_{1}, S_{2}\right) \leqslant$ $d\left(S_{1}, A^{m} x\right)+d\left(S_{2}, A^{m} x\right)<\frac{2}{3} d\left(S_{1}, S_{2}\right)$ which is absurd. Now it is clear that the set of positive integers $m \geqslant M$ for which $d\left(A^{m} x, S_{1}\right)<\frac{1}{3} d\left(S_{1}, S_{2}\right)$ is nonempty, since $\varnothing \neq S_{1} \subset \mathscr{L}(x)$. Similarly the set of positive integers $m \geqslant M$ for which $d\left(A^{m} x, S_{2}\right)<\frac{1}{3} d\left(S_{1}, S_{2}\right)$ is also nonempty. Let, for $m_{1}>M, d\left(A^{m_{1}} x, S_{1}\right)$ $<\frac{1}{3} d\left(S_{1}, S_{2}\right)$. There exist integers $n>m_{1}$ such that $d\left(A^{n} x, S_{2}\right)<\frac{1}{3} d\left(S_{1}, S_{2}\right)$. Let $k+1$ be the least such integer. Then $d\left(A^{k+1} x, S_{2}\right)<\frac{1}{3} d\left(S_{1}, S_{2}\right)$ and $d\left(A^{k} x, S_{1}\right)$ $<\frac{1}{3} d\left(S_{1}, S_{2}\right)$. We have

$$
\begin{aligned}
d\left(S_{1}, S_{2}\right) & <d\left(S_{1}, A^{k} x\right)+d\left(A^{k} x, A^{k+1} x\right)+d\left(A^{k+1} x, S_{2}\right) \\
& <\frac{1}{3} d\left(S_{1}, S_{2}\right)+\frac{1}{3} d\left(S_{1}, S_{2}\right)+\frac{1}{3} d\left(S_{1}, S_{2}\right) .
\end{aligned}
$$

This is absurd. Therefore the hypothesis that $\mathscr{L}(x)=S_{1} \cup S_{2}$ with $S_{1}$ and $S_{2}$ nonempty, disjoint, closed subsets of $\mathscr{L}(x)$ leads to a contradiction. Hence $\mathscr{L}(x)$ is connected.

By Theorem 1 in Berge [1, p. 96] it follows that $\mathscr{L}(x)$ is either a singleton or is uncountable. We have proved above that $\lim _{m \rightarrow \infty} d\left(A^{m} x, \mathscr{L}(x)\right)=0$, so that 
when $\mathscr{L}(x)$ is a singleton $\{\xi\}$, say, $\lim _{m \rightarrow \infty} d\left(A^{m} x, \xi\right)=0$. Thus $\lim _{m \rightarrow \infty} A^{m} x=$ $\xi \in F(A)$.

To prove that $\mathscr{L}(x)$, if uncountable, lies on the boundary of $F(A)$, we observe that in this case $A^{k} x \notin F(A), k=0,1,2, \ldots$ If $\xi \in \mathscr{L}(x) \subset F(A)$ is an interior point of $F(A), A^{k} x \in F(A)$ for some $k$, as $F(A)$ is a neighborhood of $\xi$ and some subsequence of $\left\{A^{m} x\right\}$ converges to $\xi$. This is a contradiction.

Remark 1. We observe here that Theorem 2 of Diaz and Metcalf [6] is a corollary of our theorem if we replace $\varphi$ by $d$. For this we have only to show that when $\mathscr{L}(x)$ is nonempty, the assumptions of Diaz and Metcalf imply the compactness of $\bar{O}(x, A)$. This has been shown in [8]. The following example shows that our theorem is indeed a generalization of the theorem of Diaz and Metcalf.

Take $X=\{a, b, c, d, e\}$ with the metric $d(x, y)=1$ if $x \neq y$ and $d(x, y)=0$ if $x=y$. Take the mapping $A: X \rightarrow X$ such that $A a=b, A b=c, A c=d, A d=e$ $=A e$. Here $F(A)=\{e\}$ and it is easy to see that for $x \neq A x, d(A x, F(A))<$ $d(x, F(A))$ is not satisfied for $x=a, b$ or $c$. Therefore we cannot invoke the theorem of Diaz and Metcalf to show that $\mathscr{L}(a)$ is a closed, connected subset of $F(A)$. We now define a function $\varphi: X \times X \rightarrow R_{0}$ the schematic representation of which is given by

\begin{tabular}{c|c|c|c|c|c} 
& $a$ & $b$ & $c$ & $d$ & $e$ \\
\hline$a$ & 0 & 4 & 5 & 9 & 10 \\
\hline$b$ & 40 & 0 & 3 & 6 & 8 \\
\hline$c$ & 50 & 30 & 0 & 2 & 7 \\
\hline$d$ & 90 & 60 & 20 & 0 & 1 \\
\hline$e$ & 100 & 80 & 70 & 10 & 0
\end{tabular}

where the value of $\varphi(x, y)$ occurs at the intersection of the row containing $x$ with the column containing $y$. We have

$$
\begin{aligned}
1 & =\varphi(d, F(A))=\varphi(A c, F(A))<\varphi(c, F(A))=7 \\
& =\varphi(A b, F(A))<\varphi(b, F(A))=8=\varphi(A a, F(A)) \varphi(a, F(A))=10 .
\end{aligned}
$$

Further $\varphi$ is continuous on $X \times X$ because it has the discrete topology. Also $F(A)$ is nonempty and compact and so is $\bar{O}(a, A)$. Thus we may invoke our theorem to show that $\mathscr{L}(a)$ is a nonempty, compact and connected subset of $F(A)$.

RemarK 2. Suppose, in addition to the hypotheses of Theorem 1, that $F(A)$ is an at most countable set. In this case $\lim _{m \rightarrow \infty} A^{m} x$ exists and belongs to $F(A)$, because $\mathscr{L}(x)$ is a singleton here and $\lim _{m \rightarrow \infty} d\left(A^{m} x, \mathscr{L}(x)\right)=0$. 
Corollary 1. Let $A: X \rightarrow X$ be such that $A^{k}$ is continuous for some $k$. Suppose

(i) $F\left(A^{k}\right)$ is nonempty and compact,

(ii) for each $x \in X, \bar{O}\left(x, A^{k}\right)$ is compact,

(iii) there exists a continuous real-valued function $\varphi: X \times X \rightarrow R_{0}$ such that for $x \in X-F\left(A^{k}\right), \varphi\left(A^{k} x, F\left(A^{k}\right)\right)<\varphi\left(x, F\left(A^{k}\right)\right)$.

Then, for $x \in X$, the set $\mathscr{L}_{k}(x)$ of subsequential limit points of the sequence of iterates $\left\{A^{m k} x\right\}_{m=1}^{\infty}$ is a nonempty, compact and connected subset of $F\left(A^{k}\right)$. Further the set $\mathscr{L}_{1}(x)$ of subsequential limit points of the sequence of iterates $\left\{A^{m} x\right\}_{m=1}^{\infty}$ is the union of the $k$ nonempty, compact, connected subsets $\mathscr{L}_{k}\left(A^{j} x\right), j=0,1,2, \ldots, k$ -1 .

The proof is omitted because it is a minor modification of the proof of Theorem $2^{k}$ of Diaz and Metcalf [6].

Corollary 2. Suppose, in addition to the hypotheses of Corollary 1, that $F\left(A^{k}\right)$ is an at most countable set. Then for $x \in X, \mathscr{L}_{1}(x)$ contains at most $k$ points. This is because each $\mathscr{L}_{k}\left(A^{j} x\right)$ is a singleton.

Dotson [7] calls a mapping $A$ quasi-nonexpansive if $F(A) \neq \varnothing$ and for each $x \in X-F(A), p \in F(A), d(A x, p) \leqslant d(x, p)$. We call $A$ quasi-contractive if the strict inequality sign holds. The concept of quasi-contractiveness has been discussed by Diaz and Metcalf [6]. We define the mapping $A$ to be $\varphi$-quasi-nonexpansive if $F(A) \neq \varnothing$ and for $x \in X-F(A), p \in F(A)$ we have $\varphi(A x, p) \leqslant$ $\varphi(x, p)$ where $\varphi: X \times X \rightarrow R_{0}$. We say that $A$ is $\varphi$-quasi-contractive if the strict inequality sign holds. In this connection we now prove

Theorem 2. Let $A: X \rightarrow X$ be a continuous self-map of a metric space $(X, d)$. Suppose that $A$ is $\varphi$-quasi-contractive, where $\varphi$ is a continuous function from $X \times X \rightarrow R_{0}$. Then $\mathscr{L}(x) \subset F(A)$. If $\varphi(x, y)=0 \Leftrightarrow x=y$, then $\mathscr{L}(x)$ consists of at most one point. If $\bar{O}(x, A)$ is compact in addition, then $\lim _{m \rightarrow \infty} A^{m} x$ exists and belongs to $F(A)$.

Proof. If $\mathscr{L}(x)$ is empty there is nothing to prove. Therefore we shall assume that $\mathscr{L}(x) \neq \varnothing$ and that $A^{k} x \notin F(A), k=0,1,2, \ldots$, as in Theorem 1 . Then for any $p \in F(A)$, the sequence of positive numbers $\left\{\varphi\left(A^{n} x, p\right)\right\}$ is monotonically decreasing, because $\varphi\left(A^{n+1} x, p\right)<\varphi\left(A^{n} x, p\right)$ by hypothesis. Hence $\lim _{n \rightarrow \infty} \varphi\left(A^{n} x, p\right)$ exists and is $r \geqslant 0$. Let $\xi \in \mathscr{L}(x)$ and let the subsequence $\left\{A^{n_{i}} x\right\}_{i=1}^{\infty}$ converge to $\xi$. If possible, let $\xi \neq A \xi$. Now 


$$
\begin{aligned}
r & =\lim _{i \rightarrow \infty} \varphi\left(A^{1+n_{i}} x, p\right)=\varphi\left(\lim _{i \rightarrow \infty} A^{1+n_{i}} x, p\right)=\varphi(A \xi, p) \\
& <\varphi(\xi, p)=\varphi\left(\lim _{i \rightarrow \infty} A^{n_{i}} x, p\right)=\lim _{i \rightarrow \infty} \varphi\left(A^{n_{i}} x, p\right)=\lim _{i \rightarrow \infty} \varphi\left(A^{n} x, p\right)=r .
\end{aligned}
$$

This contradiction proves that $\xi=A \xi$ and so $\xi \in F(A)$. We have thus proved that $\mathscr{L}(x) \subset F(A)$. Obviously $\mathscr{L}(x)$ is closed.

Assume now that $\varphi(x, y)=0$ if and only if $x=y$. Let $p, q \in \mathscr{L}(x) \subset F(A)$. Obviously $\varphi\left(A^{m} x, p\right) \rightarrow 0$ as $m \rightarrow \infty$. If the subsequence $\left\{A^{m_{i}} x\right\}_{i=1}^{\infty}$ converges to $q$, then $\varphi\left(A^{m_{i}} x, p\right) \rightarrow \varphi(p, q)$. Hence $\varphi(q, p)=0$ so that $p=q$. Therefore $\mathscr{L}(x)$ can consist of at most one point. If $\bar{O}(x, A)$ is compact, then $\mathscr{L}(x)$ is obviously nonempty and so is a singleton. Let $\mathscr{L}(x)=\{p\}$. If $A^{n} x \nrightarrow p$ as $n \rightarrow \infty$ then for some $\varepsilon>0$ there exists a subsequence $\left\{A^{n_{i}} x\right\}_{i=1}^{\infty}$ with $d\left(A^{n_{i}} x, p\right) \geqslant \varepsilon>0$. The compactness of $\bar{O}(x, A)$ implies the existence of a subsequence of $\left\{A^{n_{i}} x\right\}_{i=1}^{\infty}$ converging to $p$ as $\mathscr{L}(x)=\{p\}$. This contradicts our hypothesis that $d\left(A^{n_{i}} x, p\right) \geqslant \varepsilon$. Hence $d\left(A^{n} x, p\right) \rightarrow 0$ as $n \rightarrow \infty$, implying $A^{n} x$ $\rightarrow p$.

Browder and Petryshyn [2, 3] define a self-map $A$ of a Banach space to be asymptotically regular if $A^{n+1} x-A^{n} x \rightarrow 0$ strongly as $n \rightarrow \infty$. We shall say that a mapping $A$ is $\varphi$-asymptotically regular if $\varphi\left(A^{n} x, A^{n+1} x\right) \rightarrow 0$ as $n \rightarrow \infty$. We are now in a position to give our

THEOREM 3. Let $A$ be a continuous self-map of a metric space $(X, d)$. Suppose

(i) $F(A)$ is nonempty and compact,

(ii) there exists a continuous function $\varphi: X \times X \rightarrow R_{0}$ such that $\varphi(y, z)=0$ if and only if $y=z$,

(iii) $A$ is $\varphi$-asymptotically regular,

(iv) $\bar{O}(x, A)$ is compact.

Then $\mathscr{L}(x)$ is a nonempty, compact and connected subset of $F(A)$. Either $\mathscr{L}(x)$ is singleton or uncountable. In the case $\mathscr{L}(x)$ is a singleton $\lim _{n \rightarrow \infty} A^{n} x$ exists and belongs to $F(A)$. In the case $\mathscr{L}(x)$ is uncountable it is contained in the boundary of $\boldsymbol{F}(\boldsymbol{A})$.

Proof. If $A^{k} x \in F(A)$ for some $k$, then the proof is trivial. Therefore, assume $A^{k} x \notin F(A)$ for all $k$. The sequence $\left\{\varphi\left(A^{m} x, F(A)\right)\right\}_{m=1}^{\infty}$ is non-increasing and bounded below by zero and so converges to $r \geqslant 0$. Since $\bar{O}(x, A)$ is compact, $\mathscr{L}(x) \neq \varnothing$. Let $\xi \in \mathscr{L}(x)$ with $A^{m_{i}} x \rightarrow \xi$ as $i \rightarrow \infty$. Then $\varphi\left(A^{m_{i}} x, A^{1+m_{i}} x\right) \rightarrow$ $\varphi(\xi, A \xi)=0$, since $A$ is $\varphi$-asymptotically regular. Hence $\xi=A \xi$ and $\xi \in F(A)$. Therefore $\mathscr{L}(x) \subset F(A)$. Obviously $\mathscr{L}(x)$ is closed. Since $F(A)$ is compact and $\mathscr{L}(x)$ is closed, $\mathscr{L}(x)$ itself is compact. In view of the proof of Theorem 1 , to 
prove that $\mathscr{L}(x)$ is connected we need prove only $d\left(A^{m} x, F(A)\right) \rightarrow 0$ as $m \rightarrow \infty$ and this follows from the compactness of $\bar{O}(x, A)$ and the fact that $\mathscr{L}(x) \subset F(A)$. The remaining part of the proof is as in Theorem 1.

We may relax the compactness conditions on $F(A)$ by assuming $A$ to be $\varphi$-quasi-nonexpansive. This we state as

THEOREM 4. Let $A$ be a continuous self-map of a metric space $(X, d)$. Suppose

(i) $F(A)$ is nonempty,

(ii) $A$ is $\varphi$-asymptotically regular where $\varphi$ is a continuous function: $X \times X \rightarrow R_{0}$ and $\varphi(y, z)=0$ if and only if $y=z$.

Then $\mathscr{L}(x) \subset F(A)$. If, in addition, $A$ is $p$-quasi-nonexpansive, then $\mathscr{L}(x)$ consists of at most one point. If $\bar{O}(x, A)$ is compact, then $\lim _{m \rightarrow \infty} A^{n} x=p$, where $\mathscr{L}(x)=\{p\}$

Proof. The fact that $A$ is $\varphi$-asymptotically regular and vanishes only on the diagonal shows that $\mathscr{L}(x) \subset F(A)$. If $A$ is $\varphi$-quasi-nonexpansive and $p, q \in \mathscr{L}(x)$ with $p \neq q$, then $\varphi\left(A^{n} x, q\right) \rightarrow r \geqslant 0$. Also there exist subsequences $\left\{A^{m_{i}} x\right\}$, $\left\{A^{n_{j}} x\right\}$ such that $A^{m_{i}} x \rightarrow p, A^{n_{j}} \rightarrow q$. Hence $\varphi\left(A^{m_{i}} x, A^{n_{j}} x\right) \rightarrow \varphi(p, q)$. Keeping $i$ fixed and letting $j \rightarrow \infty$, we have $\varphi\left(A^{m_{i}} x, A^{n_{j}} x\right) \rightarrow \varphi\left(A^{m_{i}} x, q\right)$. We can extract a subsequence $\left\{m_{j}^{\prime}\right\}$ from $\left\{m_{i}\right\}$ such that $m_{j}^{\prime}>n_{j}$. Since $\lim _{i \rightarrow \infty} \varphi\left(A^{m_{i}} x, q\right)=r$ $\geqslant 0$, we have,

$$
r=\lim _{i \rightarrow \infty} \varphi\left(A^{m_{i}} x, q\right)=\lim _{m_{j}^{\prime} \rightarrow \infty} \varphi\left(A^{m_{j}^{\prime}}, q\right) \leqslant \lim _{n_{j} \rightarrow \infty} \varphi\left(A^{n_{j} x}, q\right)=\varphi(q, q)=0 .
$$

But $\varphi\left(A^{m_{i}} x, q\right) \rightarrow \varphi(p, q)$. Therefore $\varphi(p, q)=0$ whence $p=q$. Thus $\mathscr{L}(x)$ consists of at most one point. If $\bar{O}(x, A)$ is compact, then $\mathscr{L}(x)$ is nonempty and so $\mathscr{L}(x)=\{p\}$, say. Now, proceeding as in Theorem 2 we can show that $\lim _{n \rightarrow \infty} A^{n} x=p$.

We now take the range of $A$ to be compact and derive

Theorem 5. Let $A: X \rightarrow X$ be continuous. Suppose

(i) $A(X)$ is compact,

(ii) $A$ is $\varphi$-asymptotically regular where $\varphi$ is a continuous function: $X \times X \rightarrow R_{0}$ and $\varphi(x, y)=0$ if and only if $x=y$.

Then, for $x \in X$, the set $\mathscr{L}(x)$ is a nonempty, compact and connected subset of $F(A)$. Either $\mathscr{L}(x)$ contains exactly one point or is uncountable. In the case $\mathscr{L}(x)$ is a singleton, $\lim _{m \rightarrow \infty} A^{m} x$ exists and belongs to $F(A)$. In the case $\mathscr{L}(x)$ is uncountable, it is contained in the boundary of $F(A)$.

Proof. Since $A$ is continuous, $F(A)$ is closed and so is compact as $F(A) \subset$ $A(X)$, which is compact. Since $\bar{O}(A x, A) \subset A(X)$, we have $\bar{O}(A x, A)$ is compact. 
Condition (ii) now implies that $\mathscr{L}(A x) \subset F(A)$. But $\mathscr{L}(x)=\mathscr{L}(A x)$. Hence $\mathscr{L}(x) \subset F(A)$. The compactness of $\bar{O}(A x, A)$ implies that $d\left(A^{n} x, F(A)\right) \rightarrow 0$ as $n \rightarrow \infty$. The remaining conclusions can be derived as in Theorem 1 .

Following the idea of Singh and Zorzitto [9] we have

THEOREM 6. Let $A$ be a continuous self-map of a metric space $(X, d)$. Suppose

(i) $F(A)$ is nonempty and compact

(ii) there exists a continuous function $\varphi: X \times X \rightarrow R_{0}$ such that $\varphi(y, z)=0$ if and only if $y=z$ and for $y \in X-F(A), \varphi(A y, F(A)) \leqslant \varphi(y, F(A))$ and $\varphi\left(A^{m} y, F(A)\right)<\varphi(y, F(A))$ for an integer $m=m(y)$,

(iii) $\bar{O}(x, A)$ is compact.

Then $\mathscr{L}(x)$ is a nonempty, compact and connected subset of $F(A)$. Either $\mathscr{L}(x)$ is a singleton or is uncountable. In the case $\mathscr{L}(x)$ is a singleton, $\lim _{m \rightarrow \infty} A^{m} x$ exists and belongs to $F(A)$. In the case $\mathscr{L}(x)$ is uncountable it is contained in the boundary of $F(A)$.

Proof. Since $\bar{O}(x, A)$ is compact, $\mathscr{L}(x)$ is nonempty. It is enough to prove that $\mathscr{L}(x) \subset F(A)$. The remaining portion of the proof can be derived as in Theorem 1. Assume $A^{k} x \notin F(A)$ for all $k$. Let $\xi \in(x)$. If possible let $\xi \neq A \xi$. Hence there exists a subsequence $\left\{A^{n_{i}} x\right\}$ of $\left\{A^{n} x\right\}$ such that $A^{n_{i}} x \rightarrow \xi$. Obviously, $\lim _{n \rightarrow \infty} \varphi\left(A^{n} x, F(A)\right)$ exists and is equal to $r \geqslant 0$. Now $r=$ $\lim _{i \rightarrow \infty} \varphi\left(A^{m(\xi)+n_{i}} x, F(A)\right) \leqslant \varphi\left(\lim _{i \rightarrow \infty} A^{m(\xi)+n_{i}} \dot{x}, F(A)\right)=\varphi\left(A_{\xi}^{m(\xi)}, \quad F(A)\right)<$ $\varphi(\xi, F(A))$. Proceeding as in Theorem 1 , we can show that $\varphi(\xi, F(A)) \leqslant r$. Thus $r \leqslant \varphi\left(A^{m(\xi)} \xi, F(A)\right)<\varphi(\xi, F(A)) \leqslant r$, which is absurd. Thus $\xi=A \xi$ and $\mathscr{L}(x)$ $\subset F(A)$.

Corresponding to Theorem 2 we state the following theorem without proof as it can be derived by combining the methods of Theorem 2 and Theorem 6 .

TheOREM 7. Let $A: X \rightarrow X$ be a continuous self-map of a metric space $(X, d)$. Suppose that $F(A) \neq \varnothing$ and $A$ is $\varphi$-quasi-inonexpansive where $\varphi: X \times X \rightarrow R_{0}$ is continuous. Assume further that for $y \in X-F(A)$ and $p \in F(A)$ there exists an integer $m=m(y, p)$ such that $\varphi\left(A^{m} y, p\right)<\varphi(y, p)$. Then for $x \in X, \mathscr{L}(x) \subset$ $F(A)$. If $\varphi(y, z)=0 \Leftrightarrow y=z$, then, for any $x \in X, \mathscr{L}(x)$ consists of at most one point. If in addition, $\bar{O}(x, A)$ is compact then $\lim _{m \rightarrow \infty} A^{m} x$ exists and belongs to $F(A)$.

Now we shall use conditions similar to those of Caristi [4] to derive the same conclusions as those of Diaz and Metcalf [6]. 
THEOREM 8. Let $A$ be a self-map of a metric space $(X, d)$. Suppose

(i) $A$ is continuous at each pont of $F(A)$,

(ii) $F(A)$ is nonempty and compact,

(iii) there exists a function $\psi: X \rightarrow R_{0}$ such that for $y \in X, d(A y, F(A))<\psi(y)$ $-\psi(A y)$.

Then, for $x \in X, \mathscr{L}(x)$ is a nonempty, compact and connected subset of $F(A)$. Either $\mathscr{L}(x)$ is a singleton or is uncountable. In the case $\mathscr{L}(x)$ is a singleton, $\lim _{m \rightarrow \infty} A^{m} x$ exists and belongs to $F(A)$. In the case $\mathscr{L}(x)$ is uncountable, it is contained in the boundary of $F(A)$.

Proof. We assume at the outset $A^{k} x \notin F(A)$ for all $k$, because otherwise the proof is trivial. This implies that $\psi\left(A^{k+1} x\right)<\psi\left(A^{k} x\right)$ for all $k$. Thus $\left\{\psi\left(A^{k} x\right)\right\}_{k=1}^{\infty}$ is a monotonically decreasing sequence of reals bounded below by zero and so converges to $r \geqslant 0$. We have

$$
\begin{aligned}
& d\left(A^{m} x, F(A)\right) \leqslant \psi\left(A^{m-1} x\right)-\psi\left(A^{m} x\right), \\
& d\left(A^{m+1} x, F(A)\right) \leqslant \psi\left(A^{m} x\right)-\psi\left(A^{m+1} x\right), \\
& \therefore\left(A^{n} x, F(A)\right) \leqslant \psi\left(A^{n-1} x\right)-\psi\left(A^{n} x\right),
\end{aligned}
$$

whence, by adding, we get

$$
\begin{gathered}
d\left(A^{m} x, F(A)\right)+d\left(A^{m+1} x, F(A)\right)+\cdots+d\left(A^{n} x, F(A)\right) \\
\leqslant \psi\left(A^{m-1} x\right)-\psi\left(A^{n} x\right) .
\end{gathered}
$$

For $m, n$ sufficiently large, the right hand side can be made less than any preassigned $\varepsilon>0$, since $\left\{\psi\left(A^{k} x\right)\right\}_{k=1}^{\infty}$ is a convergent sequence of reals. Hence $d\left(A^{m} x, F(A)\right) \rightarrow 0$ as $m \rightarrow \infty$. Now, since $F(A)$ is compact we can find a $p_{m} \in F(A)$ such that $d\left(A^{m} x, F(A)\right)=d\left(A^{m} x, p_{m}\right)$. The sequence $\left\{p_{m}\right\}_{m=1}^{\infty}$ will have a convergent subsequence $\left\{p_{m_{i}}\right\}_{i=1}^{\infty}$ converging to $p \in F(A)$. Now

$$
\begin{aligned}
d\left(p, A^{m_{i}} x\right) & \leqslant d\left(p, p_{m_{i}}\right)+d\left(p_{m_{i}}, A^{m_{i} x}\right) \\
& =d\left(p, p_{m_{i}}\right)+d\left(A^{m_{i}} x, F(A)\right) \rightarrow 0 \text { as } i \rightarrow \infty .
\end{aligned}
$$

Thus $A^{m_{i}} x \rightarrow p$ as $i \rightarrow \infty$ and so $p \in \mathscr{L}(x)$ and hence $\mathscr{L}(x)$ is nonempty. If $\xi \in \mathscr{L}(x)$, then there is a subsequence $\left\{A^{n_{i}} x\right\}_{i=1}^{\infty}$ converging to $\xi$. Now $d(\xi, F(A))=d\left(\lim A^{n_{i}} x, F(A)\right)=\lim d\left(A^{n_{i}} x, F(A)\right)=0$ and as $F(A)$ is closed (being compact), $\xi \in F(A)$. Thus $\mathscr{L}(x) \subset F(A)$. Obviously $\mathscr{L}(x)$ is a closed subset of the compact set $F(A)$ and hence is itself compact. Now we can proceed as in proving Theorem 1 to establish the remaining conclusions.

COROLlary 3. If $A^{k}$ satisfies the conditions of Theorem 8 , then the set $\mathscr{L}_{k}(x)$ of subsequential limit points of the sequence $\left\{A^{m k_{x}} x\right\}_{m=1}^{\infty}$ is a nonempty, compact, and 
connected subset of $F\left(A^{k}\right)$. The set $\mathscr{L}_{1}(x)$ of the subsequential limit points of $\left\{A^{m} x\right\}_{m=1}^{\infty}$ is the union of the $k$ closed and connected sets $\mathscr{L}_{k}\left(A^{j} x\right), j=0,1,2, \ldots, k$ - 1. If $F\left(A^{k}\right)$ is at most countable, then $\mathscr{L}_{1}(x)$ consists of at most $k$ points.

COROLlaRY 4. The conclusions of Theorem 8 remain valid if condition (iii) is replaced by

(iii)' there exists a monotonically decreasing sequence $\left\{r_{n}\right\}$ of positive reals such that $d\left(A^{n+1} x, F(A)\right) \leqslant r_{n}-r_{n+1}$, or

(iii)" there exists a sequence $\left\{s_{n}\right\}$ of positive reals converging to zero such that $d\left(A^{n} x, F(A)\right) \leqslant s_{n}$.

Tarafdar [10] has extended some results of Diaz and Metcalf [2] to uniform spaces. We shall show that the results of Tarafdar still hold when our condition replaces his inequality. Our notations will conform to those of Thron [11].

Let $(X, h)$ be a uniform space, $h$ being the uniformity. The uniform topology induced by $h$ will be denoted by $\mathscr{T}_{h}$. A family $\left\{\rho_{\alpha}: \alpha \in I\right\}$ of pseudometrics on $X$ is called an associated family for the uniformity $h$ on $X$ if the family $\{H(\alpha, \varepsilon) \mid \alpha$ $\in I, \varepsilon>0\}$ where $H(\alpha, \varepsilon)=\left\{(x, y) \mid \rho_{\alpha}(x, y)<\varepsilon\right\}$ is a subbase for $h$. A family $\left\{\rho_{\alpha} \mid \alpha \in I\right\}$ of pseudometrics on $X$ is called an augmented associated family for $h$ if $\left\{\rho_{\alpha} \mid \alpha \in I\right\}$ is an associated family for $h$ and has the additional property that given $\alpha, \beta \in I$, there is $\gamma \in I$ such that $\rho_{\gamma}(x, y) \geqslant \max \left(\rho_{\alpha}(x, y), \rho_{\beta}(x, y)\right)$ for all $x, y \in X$. An associated family and an augmented associated family for $h$ will be denoted respectively by $\mathscr{F}(h)$ and $\mathscr{F} *(h)$.

We are now in a position to give our

THEOREM 9. Let $(X, h)$ be a Hausdorff uniform space and $\mathscr{F}^{*}(h)=\left\{\rho_{\alpha} \mid \alpha \in I\right\}$. Let $A: X \rightarrow X$ be $\mathscr{T}_{h}$-continuous. Suppose

(i) $A(X)$ is $\mathscr{T}_{h}$-compact,

(ii) $A$ is $\varphi$-asymptotically regular where $\varphi$ is a $\mathscr{T}_{h} \times \mathscr{T}_{h}$ continuous function on $X \times X \rightarrow R_{0}$ such that $\varphi(x, y)=0$ if and only if $x=y$.

Then, for each $x \in X$, the $\mathscr{T}_{h}$-cluster set $\mathscr{L}(x)$ is a nonempty $\mathscr{T}_{h}$-closed and $\mathscr{T}_{h}$-connected subset of $F(A)$. In the case $\mathscr{L}(x)$ is just one point then $\mathscr{T}_{h}$-lim $A^{n} x$ exists and belongs to $F(A)$. In the case $\mathscr{L}(x)$ contains more than one point then it is contained in the $\mathscr{T}_{h}$-boundary of $F(A)$.

Proof. The sequence $\left\{A^{n} x\right\}_{n=1}^{\infty}$ being a net in $A(X)$, which is compact, $\mathscr{L}(x)$ is nonempty. If $y \in \mathscr{L}(x)$, then there is a subnet $\left\{A^{n}{ }^{n} x\right\}_{j \in J}$ of the net $\left\{A^{n} x\right\}_{n=1}^{\infty}$ such that $A^{n_{j}} \rightarrow y$ in the $\mathscr{T}_{h}$-topology. Since $A$ is $\mathscr{T}_{h}$-continuous, $A^{1+n_{j}} x \rightarrow A y$ in the $\mathscr{T}_{h}$-topology. Hence $\varphi\left(A^{n_{j} x}, A^{1+n_{j}} x\right) \rightarrow \varphi(y, A y)$ as $\varphi$ is $\mathscr{T}_{h} \times \mathscr{T}_{h}$ continuous. Since $A$ is $\varphi$-asymptotically regular, $\varphi(y, A y)=0$ and hence by condition (ii), 
$y=A y$. Therefore $\mathscr{L}(x) \subset F(A)$. Obviously $\mathscr{L}(x)$ is closed. Now, we can proceed as in Tarafdar [10, Theorem 2.1] to prove that $\mathscr{L}(x)$ is $\mathscr{T}_{h}$-connected. The other parts of the conclusion are to be established likewise.

Corresponding to Theorem 2.2 of Tarafdar [10] we have

THEOREM 10. Let $(X, h)$ be a Hausdorff uniform space and let $\left\{\rho_{\alpha} \mid \alpha \in I\right\}=$ $\mathscr{F}^{*}(h)$. Let $A: X \rightarrow X$ be $\mathscr{T}_{h}$-continuous. Suppose

(i) $F(A)$ is nonempty and compact,

(ii) there exists a $\mathscr{T}_{h} \times \mathscr{T}_{h}$ continuous function $\varphi: X \times X \rightarrow R_{0}$ such that for $y \neq A y, \varphi(A y, F(A))<\varphi(y, F(A))$,

(iii) $\bar{O}(x, A)$ is compact.

Then $\mathscr{L}(x)$ is a closed subset of $F(A)$. If $\mathscr{L}(x)$ consists of more than one point, then $\mathscr{L}(x)$ is contained in the $\mathscr{T}_{h}$-boundary of $F(A)$.

The proof is omitted. A careful perusal of the proof of Theorem 1 shows that no metric properties of the space have been used in proving that $\mathscr{L}(x)$ is a subset of $F(A)$. Therefore we have the following theorem for Hausdorff topological spaces.

THEOREM 11. Let $(X, \mathscr{T})$ be a Hausdorff topological space and $A$, a continuous self-map. Suppose

(i) $F(A)$ is nonempty and compact,

(ii) there eixsts a continuous function $\varphi: X \times X \rightarrow R_{0}$ such that for $y \neq A y$, $\varphi(A y, F(A))<\varphi(y, F(A))$.

Then $\mathscr{L}(x)$, the set cluster points of $\left\{A^{n} x\right\}_{n=1}^{\infty}$ is a closed subset of $F(A)$. If $\mathscr{L}(x)$ consists of more than one point then it is contained in the boundary of $F(A)$. If we further assume that $\varphi(x, y)=0$ if and only if $x=y$, then $\mathscr{L}(x)$ is at most a singleton. If $\mathscr{L}(x)$ is a singleton and $\bar{O}(x, A)$ is compact, then $\lim _{n \rightarrow \infty} A^{n} x$ exists and belongs to $F(A)$.

\section{References}

[1] C. Berge, Topological spaces (Oliver and Boyd, 1963).

[2] F. E. Browder and W. V. Petryshyn, 'The solution by iteration of linear functional equations in Banach spaces', Bull. Amer. Math. Soc. 72 (1966), 566-570.

[3] _ 'The solution by iteration of nonlinear functional equations in Banach spaces', Bull. Amer. Math. Soc. 72 (1966), 571-575.

[4] J. Caristi, 'Fixed point theorems for mappings satisfying inwardness conditions', Trans. Amer. Math. Soc. 215 (1976), 241-251. 
[5] J. B. Diaz and F. T. Metcalf, 'On the structure of the set of subsequential limit points of successive approximations', Bull. Amer. Math. Soc. 73 (1967), 516-519.

[6] _ 'On the set of subsequential limit points of successive approximations', Trans. Amer. Math. Soc. 135 (1969), 459-485.

[7] W. G. Dotson, Jr., 'On the Mann iterative process', Trans. Amer. Math. Soc. 149 (1970), 65-73.

[8] M. Maiti and A. C. Babu, 'On subsequential limit points of a sequence of iterates', Proc. Amer. Math. Soc. 82 (1981), 377-381.

[9] S. P. Singh and F. Zorzitto, 'On fixed point theorems in metric spaces', Ann. Soc. Sci. Bruxelles 85 (1971), 117-123.

[10] E. Tarafdar, 'An approach to fixed point theorems on uniform spaces', Trans. Amer. Math. Soc. 191 (1974), 209-225.

[11] W. J. Thron, Topological structures (Holt, Rinehart and Winston, New York, 1966).

Department of Mathematics

Indian Institute of Technology

Kharagpur, 721302

India
Department of Mathematics University College of Engineering Burla, 768018

India 